06

\title{
Терагерцевые характеристики пленок композита на основе металлоорганического перовскита и оксида графена
}

\author{
() А.В. Андрианов, А.Н. Алешин \\ Физико-технический институт им. А.Ф. Иофффе РАН, Санкт-Петербург, Россия \\ E-mail: alex.andrianov@mail.ioffe.ru
}

Поступило в Редакцию 7 апреля 2021 г.

В окончательной редакции 7 апреля 2021 г.

Принято к публикации 2 июня 2021г.

Пленки композита на основе металлоорганического перовскита $\mathrm{CH}_{3} \mathrm{NH}_{3} \mathrm{PbI}_{3}$ и частиц оксида графена (GO) с содержанием $\sim 0,2,6,15 \mathrm{wt} . \%$ исследованы методом терагерцевой спектроскопии во временно́й области в спектральном диапазоне $0.2-2.9 \mathrm{THz}$. Установлено, что коэффициент терагерцевого поглощения и действительная часть оптической проводимости композита $\mathrm{CH}_{3} \mathrm{NH}_{3} \mathrm{PbI}_{3}: \mathrm{GO}$ с ростом содержания частиц оксида графена значительно возрастают. При этом обнаружено, что вклад в поглощение и оптическую проводимость низкочастотных колебательных мод в молекуле перовскита при 0.96 и $1.92 \mathrm{THz}$ существенно уменьшается с ростом концентрации оксида графена.

Ключевые слова: металлоорганические перовскиты, оксид графена, композиционные материалы, терагерцевая спектроскопия.

DOI: 10.21883/PJTF.2021.18.51463.18815

В последние годы большое внимание в мире уделяется синтезу и исследованию свойств металлоорганических перовскитов и их композитов - новых материалов, которые находят применение в солнечных элементах (СЭ), светодиодах, полевых транзисторах и ячейках памяти [1-4]. Особенно впечатляющие результаты эти материалы демонстрируют в случае солнечных элементов, КПД которых достиг 25.5\%, что обусловлено такими их физическими свойствами, как высокий коэффициент поглощения в видимой области, большие длины диффузии и подвижности носителей заряда, а также большие времена жизни носителей [5]. Несмотря на то что были обнаружены и исследованы многочисленные новые физические свойства металлоорганических перовскитов, что привело к улучшениям параметров приборов на их основе, поведение фононов в этих материалах, в частности, в терагерцевом (THz) спектральном диапазоне $(0.1-3.0 \mathrm{THz})$ еще до конца не выяснено [6,7]. Было показано, что для металлоорганических перовскитов характерны как молекулярные колебания, связанные с органической частью молекулы, так и колебания решетки, обусловленные неорганической ее частью, из-за отсутствия химической связи между органической и неорганической частями [3]. В случае пленок металлоорганических перовскитов $\mathrm{CH}_{3} \mathrm{NH}_{3} \mathrm{PbI}_{3}$ (общепринятое обозначение $\mathrm{MAPbI}_{3}$, где $\mathrm{MA}=\mathrm{CH}_{3} \mathrm{NH}_{3}[8]$ ), полученных методом полива из раствора, в THz-диапазоне обычно наблюдаются две колебательные моды с частотами вблизи 1 и $2 \mathrm{THz}$, которые объясняются колебаниями, обусловленными изменением углов связи $\mathrm{Pb}-\mathrm{I}-\mathrm{Pb}$, и колебаниями, обусловленными изменением расстояний между атомами свинца и иода в связи $\mathrm{Pb}-\mathrm{I}$, соответственно [9].
Важным и неисследованным вопросом является влияние на фононные моды включений в $\mathrm{MAPbI}_{3}$ частиц других проводящих материалов, таких как оксид графена (graphene oxide, GO), который также применяется в фотоэлектрических устройствах [10]. Композиты на основе металлоорганических перовскитов и GO применяются при разработке новых эффективных СЭ [11-13]. Было показано, что введение малой концентрации GO в пленки $\mathrm{MAPbI}_{3}$ приводит к улучшению фотоэлектрических свойств перовскитных СЭ, а также к повышению стабильности и воспроизводимости их характеристик, так как GO - эффективный акцептор дырок, который позволяет достичь баланса подвижности электронов и дырок и увеличить эффективность разделения зарядов в активном слое СЭ. Если свойства композитов на основе металлоорганических галогенидных перовскитов и GO при низких частотах уже изучены [13], то оптические и электрические характеристики композитных пленок $\mathrm{MAPbI}_{3}: \mathrm{GO}$ в THz-области спектра до сих пор не были исследованы.

В настоящей работе исследован терагерцевый электромагнитный отклик композитных пленок на основе металлоорганического перовскита $\mathrm{MAPbI}_{3}$ и частиц GO. Измерены спектры комплексной диэлектрической проницаемости в спектральном диапазоне $0.2-2.9 \mathrm{THz}$ для композитных пленок $\mathrm{MAPbI}_{3}: \mathrm{GO}$ с содержанием $\mathrm{GO} \sim 0,2,6,15 \mathrm{wt} . \%$. Измерения были проведены с разрешением $0.1 \mathrm{THz}$ при комнатной температуре на воздухе с влажностью порядка $56 \%$.

Для приготовления образцов были использованы порошки металлоорганических перовскитов $\mathrm{MAPbI}_{3}$ с шириной запрещенной зоны $E_{g} \sim 1.55 \mathrm{eV}$, приобретенные в Xi'an Polymer Light Technology Corp. и применявшиеся без дополнительной обработки. В качестве вто- 


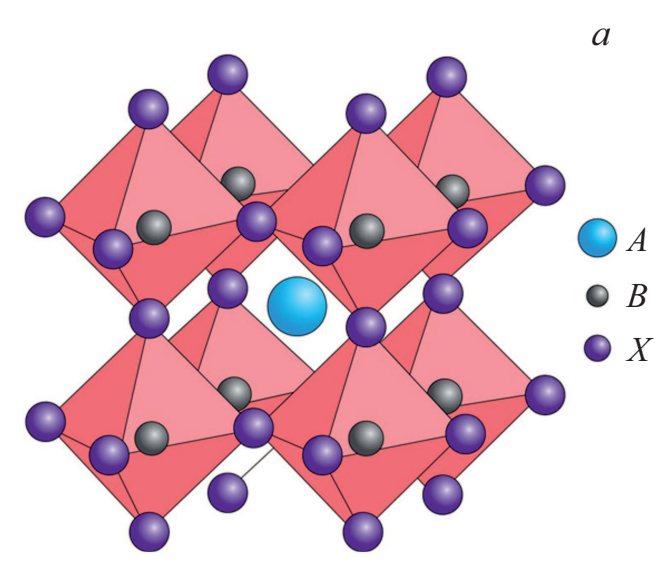

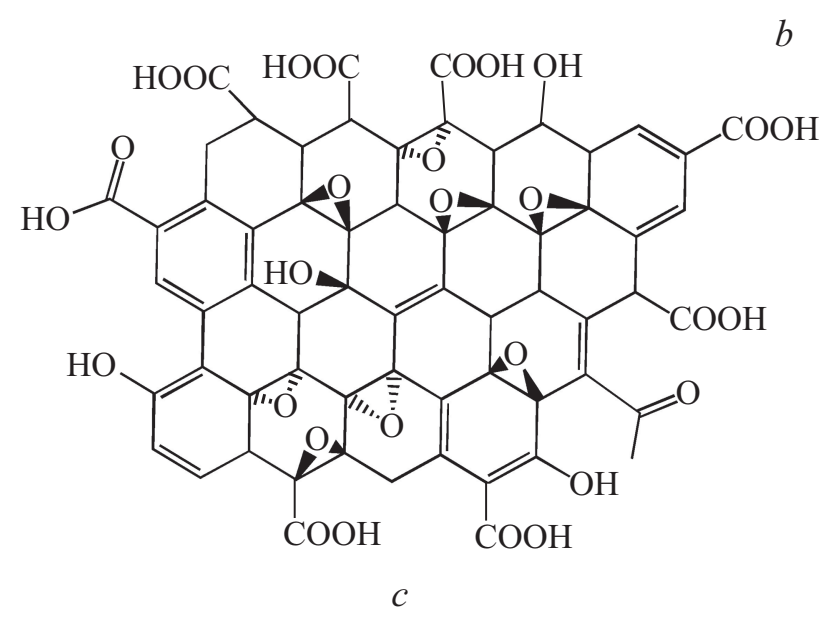

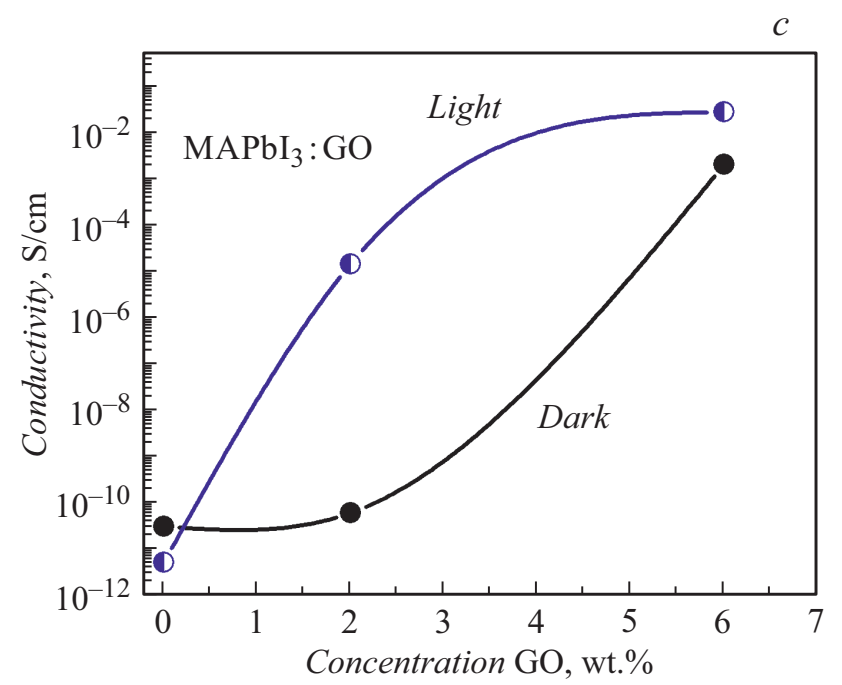

Рис. 1. Структуры металлоорганического перовскита $A^{+} B X^{-}$, где $A^{+}=\mathrm{CH}_{3} \mathrm{NH}_{3}, B=\mathrm{Pb}, X^{-}=\mathrm{I}_{3}(a)$, и оксида графена $[11]$ (b). $c$ - зависимость проводимости от концентрации GO в пленках $\mathrm{MAPbI}_{3}: \mathrm{GO}$ при $290 \mathrm{~K}$ в темноте и при освещении имитатором солнечного света.

рого компонента композитов были выбраны частицы GO, приобретенные в OOO „АкКо Лаб“. Частицы GO представляли собой чешуйки диаметром 400-500 nm, число одноатомных слоев в которых составляло от 2 до 10. Порошки $\mathrm{MAPbI}_{3}$ и частицы GO соответственно растворялись и диспергировались в полярном растворителе DMF (N,N-Dimethylformamide, $\mathrm{C}_{3} \mathrm{H}_{7} \mathrm{NO}$ ). Полученные растворы смешивались в различных весовых пропорциях, а смеси диспергировались ультразвуком в течение $10 \mathrm{~min}$ с помощью ультразвуковой мешалки Bandelin Sonopuls HD 2070 на частоте $20 \mathrm{kHz}$. Растворы $\mathrm{MAPbI}_{3}: \mathrm{GO}$ наносились в виде пленок на кремниевые подложки КДБ-10 (100) методом полива (три слоя по $50 \mu 1)$. Пленки $\mathrm{MAPbI}_{3}$ :GO высушивались при температуре $100^{\circ} \mathrm{C}$ в течение $15 \mathrm{~min}$ для удаления растворителя. Толщина пленок $\mathrm{MAPbI}_{3}: \mathrm{GO}$ составляла $2-3 \mu \mathrm{m}$. Образцы хранились в вакуумном эксикаторе до начала измерений. Были приготовлены и исследованы пленки композитов $\mathrm{MAPbI}_{3}: \mathrm{GO}$ с различным соотношением компонентов $\mathrm{MAPbI}_{3}$ и GO: $100 \% \mathrm{MAPbI}_{3}$ и 0.98:0.02, 0.94:0.06, 0.85:0.15.
На рис. 1 показаны структуры $\mathrm{MAPbI}_{3}$ (a) и GO (b). Вольт-амперные характеристики образцов на низких частотах измерялись в сандвич-геометрии по двухзондовой схеме при $290 \mathrm{~K}$ в темноте и при облучении имитатором солнечного света с использованием автоматизированной установки на основе пикоамперметра Keithley 6487. Результаты измерений зависимостей проводимости пленок $\mathrm{MAPbI}_{3}: \mathrm{GO}$ от концентрации GO в композите показаны на рис. 1,c. Как видно из рис. 1, $c$, значительная фотопроводимость композитных пленок $\mathrm{MAPbI}_{3}$ :GO на низких частотах наблюдается при малых концентрациях GO $\sim 1-6$ wt.\%. При дальнейшем увеличении концентрации GO происходит перколяционный переход в системе $\mathrm{MAPbI}_{3}: \mathrm{GO}$, и фоточувствительность композитных пленок падает.

Исследования THz-характеристик пленок композита на основе металлоорганического перовскита $\mathrm{MAPbI}_{3}$ и GO ( $\left.\mathrm{MAPbI}_{3}: \mathrm{GO}\right)$ были проведены методом THz-спектроскопии во временно́й области (THz-TDS-метод) в спектральном диапазоне $0.2-2.9 \mathrm{THz}$. THz-TDS-метод основан на генерации и детектировании широкопо- 


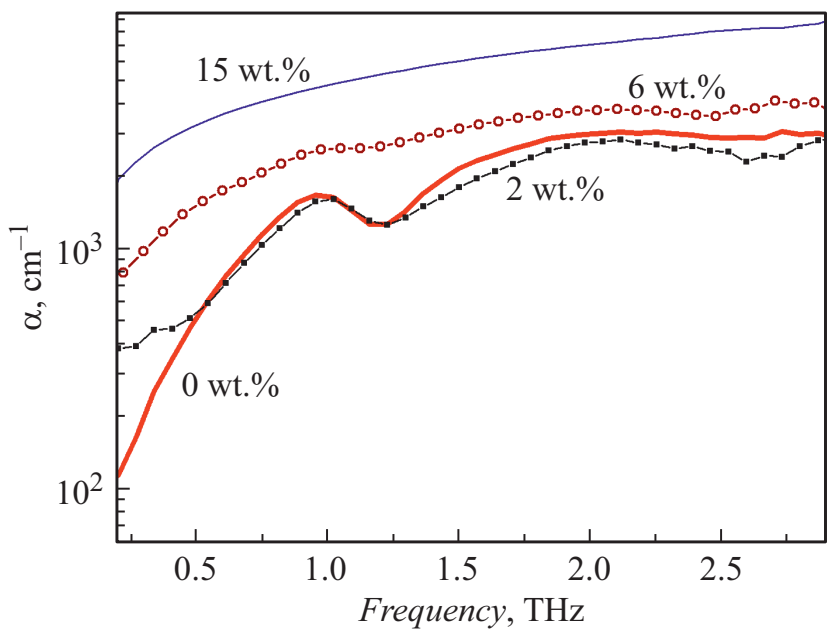

Pис. 2. Спектры коэффициента THz-поглощения по мощности для композитов $\mathrm{MAPbI}_{3}: \mathrm{GO}$ при изменении содержания $\mathrm{GO}$ от 0 до $15 \mathrm{wt} . \%$.

лосного THz-излучения с помощью фемтосекундных импульсов титан-сапфирового лазера [14]. Основное преимущество THz-TDS-метода в сравнении с методиками THz-спектроскопии, основанными на использовании фурье-спектрометров, тепловых источников и криогенных детекторов изучения, заключается в том, что все компоненты THz-TDS-системы работают при комнатной температуре, а соотношение сигнал/шум по амплитуде сигналов может достигать $10^{4}$ (и $10^{8}$ по мощности) (см., например, $[15,16])$. В настоящей работе использовалась THz-TDS-установка, детально описанная в [15]. При этом применялся дифференциальный вариант THz-TDS-методики, разработанный для исследования THz-характеристик тонких пленок на толстых подложках [15]. Измерения спектров амплитуды и фазы комплексного коэффициента THz-дифференциального пропускания образца с исследуемой тонкой пленкой позволяют прямо получить $\mathrm{THz}$-спектры действительной $\left(\varepsilon^{\prime}\right)$ и мнимой $\left(\varepsilon^{\prime \prime}\right)$ частей комплексной диэлектрической проницаемости материала пленки. Спектры коэффициента THz-поглощения по мощности $\alpha(f)$ и действительной части высокочастотной (оптической) проводимости $\sigma_{r}(f)$ исследуемого материала могут быть получены из спектров величин $\varepsilon^{\prime}$ и $\varepsilon^{\prime \prime}$ с использованием известных соотношений (см., например, [17]):

$$
\begin{gathered}
\alpha(f)=\frac{4 \pi f}{c \sqrt{2}} \frac{\varepsilon^{\prime \prime}}{\sqrt{\varepsilon^{\prime}+\sqrt{\varepsilon^{\prime 2}+\varepsilon^{\prime \prime 2}}}}, \\
\sigma_{r}(f)=2 \pi f \varepsilon_{0} \varepsilon^{\prime \prime}
\end{gathered}
$$

где $\varepsilon_{0}$ - электрическая постоянная.

На рис. 2 приведены спектры коэффициента THzпоглощения по мощности для набора исследованных композитов $\mathrm{MAPbI}_{3}: \mathrm{GO}$. Видно, что спектры поглощения сильно модифицируются при сравнительно малом изменении содержания GO. В целом поглощение значительно возрастает при увеличении содержания проводящего компонента. При этом абсолютная величина вклада в поглощение полос при 0.96 и $1.92 \mathrm{THz}$, связанных с возбуждением низкочастотных колебательных мод в молекулах перовскита $\mathrm{MAPbI}_{3}$, ранее исследованных в ряде работ (см., например, $[9,16])$, существенно падает с ростом содержания GO в композите, причем значительное ослабление вклада в поглощение низкочастотных мод при 0.96 и $1.92 \mathrm{THz}$ происходит при малой добавке GO в композит (всего 6 wt.\%, как видно из рис. 2). Вполне вероятно, что частицы GO, так же как и молекулы эфира целлюлозы [18], гасят низкочастотные колебательные моды в молекулах перовскита.

На рис. 3 приведены THz-спектры действительной части высокочастотной проводимости исследованных композитов $\mathrm{MAPbI}_{3}: \mathrm{GO}$. Проводимость композитов растет с ростом содержания GO. Здесь также можно видеть сильное изменение характера спектров при сравнительно небольшом изменении содержания GO (0-15 wt.\%). Вклад в высокочастотную проводимость колебательных мод при 0.96 и $1.92 \mathrm{THz}$ в молекулах перовскита также значительно ослабевает при сравнительно малой добавке GO в композит. Для композита с содержанием GO порядка 15 wt.\% проводимость практически не зависит от частоты, что характерно для проводимости металлического типа.

Отметим, что стабильность (и воспроизводимость) $\mathrm{THz}$-характеристик композитных пленок $\mathrm{MAPbI}_{3}: \mathrm{GO}$ хорошо коррелирует со стабильностью этих же пленок при низких частотах, что важно для приборных приложений.

Таким образом, методом терагерцевой спектроскопии во временно́й области исследован терагерцевый электромагнитный отклик композитных пленок на основе металлоорганического перовскита $\mathrm{MAPbI}_{3}$ и частиц GO. Измерены спектры комплексной диэлектрической проницаемости в спектральном диапазоне $0.2-2.9 \mathrm{THz}$

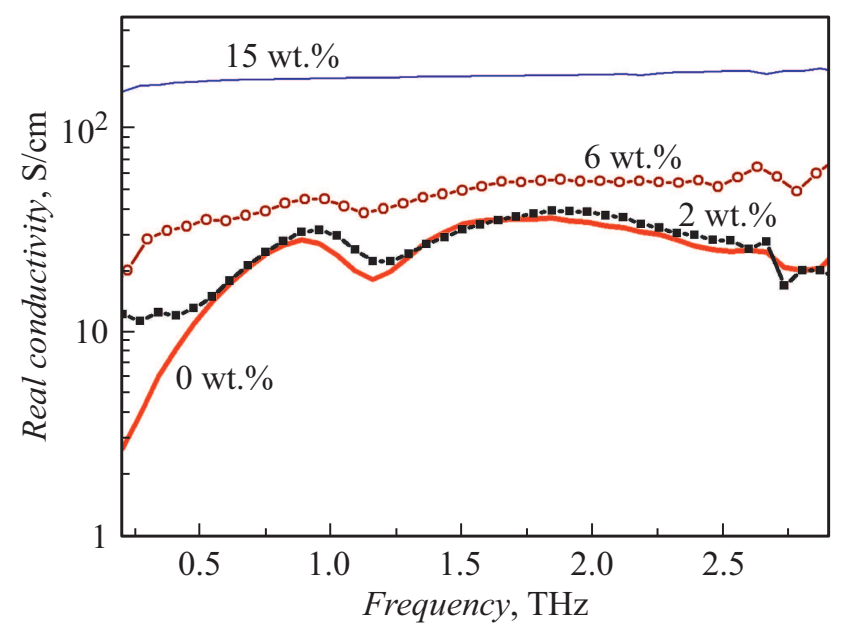

Рис. 3. Частотная зависимость действительной части комплексной проводимости композитов $\mathrm{MAPbI}_{3}: \mathrm{GO}$ при различном содержании GO. 
для композитных пленок $\mathrm{MAPbI}_{3}: \mathrm{GO}$ с содержанием $\mathrm{GO} \sim 0,2,6,15$ wt.\%. Обнаружено значительное увеличение коэффициента THz-поглощения и действительной части проводимости композита $\mathrm{MAPbI}_{3}: \mathrm{GO}$ с ростом содержания частиц GO в композите. При этом установлено, что вклад в поглощение и высокочастотную проводимость колебательных мод в молекуле перовскита при 0.96 и $1.92 \mathrm{THz}$ существенно уменьшается с ростом концентрации GO. Результаты свидетельствуют о том, что добавки GO в перовскит $\mathrm{MAPbI}_{3}$ дают возможность существенно модифицировать характеристики материала в THz-области спектра.

\section{Благодарности}

Авторы выражают благодарность А.В. Архипову за помощь в измерении проводимости образцов на постоянном токе.

\section{Конфликт интересов}

Авторы заявляют, что у них нет конфликта интересов.

\section{Список литературы}

[1] P. Wang, Y. Zhao, T. Wang, Appl. Phys. Rev., 7, 031303 (2020). https://doi.org/10.1063/5.0013912

[2] E.J. Yoo, M. Lyu, J.-H. Yun, C.J. Kang, Y.J. Choi, L. Wang, Adv. Mater., 27, 6170 (2015). https://doi.org/10.1002/adma.201502889

[3] Organic-inorganic halide perovskite photovoltaics, ed by N.-G. Park, M. Grätzel, T. Miyasaka (Springer, 2016). https://doi.org/10.1007/978-3-319-35114-8_4

[4] J. Sun, J. Wu, X. Tong, F. Lin, Y. Wang, Z.M. Wang, Adv. Sci., 5, 1700780 (2018). DOI: 10.1002/advs.201700780

[5] L.K. Ono, Y. Qi, J. Phys. D: Appl. Phys., 51, 093001 (2018). https://doi.org/10.1088/1361-6463/aacb6e

[6] Y.M. Lee, J.-H. Yun, A. Matsuyama, S. Kobon, I. Maeng, M. Lyu, Appl. Phys. Express, 12, 051003 (2019). https://doi.org/10.7567/1882-0786/ab0eec

[7] I. Maeng, Y.M. Lee, J. Park, S.R. Raga, C. Kang, C.-S. Kee, B.D. Yu, S. Hang, L.K. Ono, Y. Qi, M.-C. Jung, M. Nakamura, Sci. Rep., 9, 5811 (2019). https://doi.org/10.1038/s41598-019-42359-8

[8] H.S. Jung, N.-G. Park, Small, 11, 10 (2015). https://doi.org/10.1002/smll.201402767

[9] C. La-o-vorakit, J. Kadro, T. Salim, D. Zhac, T. Ahmed, Y.M. Lam, J.X. Zhu, R.A. Marcus, M.-E. Michel-Beyerke, E.E.M. Chia, J. Phys. Chem. Lett., 7, 1 (2016). https://doi.org/10.1021/acs.jpclett.5b02223

[10] F. Bonaccorso, Z. Sun, T. Hasan, A.C. Ferrari, Nature Photon., 4, 611 (2010). DOI: 10.1038/nphoton.2010.186

[11] J.S. Yeo, R. Kang, S. Lee, Y.J. Jeon, N. Myoung, C.L. Lee, D.Y. Kim, J.M. Yun, Y.H. Seo, S.S. Kim, S.I. Na, Nano Energy, 12, 96 (2015). http://dx.doi.org/10.1016/j.nanoen.2014.12.022

[12] J.K. Kim, S.J. Kim, M.J. Park, S. Bae, S.-P. Cho, Q.G. Du, D.H. Wang, J.H. Park, B.H. Hong, Sci. Rep., 5, 14276 (2015). DOI: $10.1038 /$ srep14276

[13] C.-C. Chung, S. Narra, E. Jokar, H.-P. Wu, E.W.-G. Diau, J. Mater. Chem. A, 5, 13957 (2017). https://doi.org/10.1039/C7TA04575A
[14] J. Neu, C.A. Schmuttenmaer, J. Appl. Phys., 124, 231101 (2018). https://doi.org/10.1063/1.5047659

[15] A.V. Andrianov, A.N. Aleshin, V.N. Truhin, A.V. Bobylev, J. Phys. D: Appl. Phys., 44, 265101 (2011). https://doi.org/10.1063/1.5047659

[16] А.В. Андрианов, А.Н. Алешин, Л.Б. Матюшкин, Письма в ЖЭТФ, 109 (1-2), 30 (2019). https://doi.org/10.1134/S0370274X19010065

[17] М. Борн, Э. Вольф, Основы оптики (Наука, М., 1975), c. 570.

[18] А.В. Андрианов, А.Н. Алешин, Письма в ЖТФ, 46 (10), 51 (2020). DOI: 10.21883/PJTF.2020.10.49434.18269 